\title{
Quasistationary High Confinement Discharges with trans-Greenwald Density on TEXTOR-94
}

\author{
G. Mank, ${ }^{1}$ A. M. Messiaen, ${ }^{2}$ J. Ongena, ${ }^{2}$ B. Unterberg, ${ }^{1}$ P. Dumortier, ${ }^{2}$ K. H. Finken, ${ }^{1}$ R. Jaspers, ${ }^{3}$ H. R. Koslowski, ${ }^{1}$ \\ A. Krämer-Flecken, ${ }^{1}$ J. Rapp, ${ }^{1}$ U. Samm,${ }^{1}$ G. van Wassenhove, ${ }^{2}$ R. R. Weynants, ${ }^{2}$ and TEXTOR-94 Team ${ }^{1,2,3}$ \\ ${ }^{1}$ Institut für Plasmaphysik, Forschungszentrum Jülich GmbH, EURATOM Association, D-52425 Jülich, Germany \\ ${ }^{2}$ Laboratoire de Physique de Plasmas/Laboratorium voor Plasmafysica, Ecole Royale Militaire/Koninklijke Militaire School, \\ EURATOM Association, B-1000 Brussels, Belgium \\ ${ }^{3}$ FOM Instituut voor Plasmafysica Rijnhuizen, EURATOM Association, NL-3430 BE Nieuwegein, The Netherlands
}

(Partners in the Trilateral Euregio Cluster)

(Received 31 March 2000)

\begin{abstract}
Confinement quality as good as ELM-free H-mode at densities substantially above the Greenwald density limit $\left(\bar{n}_{e, 0} / n_{\mathrm{GW}}=1.4\right)$ has been obtained in discharges with a radiative boundary under quasistationary conditions for 20 times the energy confinement time. This is achieved by optimizing the gas-fueling rate of RI-mode discharges which tailors their favorable energy confinement and leads to discharges with beta values just below the operational limit $\beta_{n}=2$ of TEXTOR-94, thereby effectively avoiding confinement back transitions or disruptions. In addition, this high-density regime is favorable for helium removal and results in figures of merit $\tau_{p, H e}^{*} / \tau_{E} \approx 10-15$, relevant for a future fusion power reactor.
\end{abstract}

PACS numbers: 52.55.Fa, 52.40.Hf, 52.25.Fi, 52.25.Vy

A subject of intense investigations in present day fusion research is the development of an operational scenario, providing at the same time (i) confinement with at least H-mode quality and (ii) high densities around or above the empirical Greenwald density value $n_{\mathrm{GW}}=I_{P} / \pi a^{2}$ [1] [with $I_{P}$ the plasma current (MA), the minor plasma radius $(\mathrm{m})$, and $n_{\mathrm{GW}}$ in units $10^{20} \mathrm{~m}^{-3}$ ]. Success in this direction has been obtained in the past years on limiter $[2,3]$ and divertor tokamaks by pellet injection or adjusting deuterium fueling and pumping [4,5]. Recently, high energy confinement at a Greenwald number $\bar{n}_{e, 0} / n_{\mathrm{GW}}=1.4$ ( $\bar{n}_{e, 0}$ being the central averaged chord density) was obtained at DIII-D by optimizing divertor pumping in gas puffed $\mathrm{H}$-mode discharges with edge localized modes (ELM) [6,7]. This is in contrast to most H-mode discharges which undergo a confinement degradation when approaching this value [8]. In this paper we show that it is possible in addition to substantially overcome the Greenwald limit under quasistationary conditions with confinement of ELM-free H-mode quality, high $\beta$, $q=3.4$, and a regime favorable for heat and particle exhaust. This is obtained by optimizing the gas-fueling rate in radiatively improved (RI) mode plasmas on the limiter tokamak TEXTOR-94 in order to maintain a sufficiently low neutral pressure in the scrape off layer (SOL).

The RI-mode on TEXTOR-94 is obtained with edge radiation cooling due to neon seeding under boronized wall conditions or sputtering of silicon under siliconized wall conditions. A careful adjustment of the edge and heating conditions is necessary to reach RI-mode confinement, proportional to the electron density, leading to high confinement at high density. The confinement improvement observed in the RI-mode has been interpreted as resulting from the suppression of ion thermal gradient (ITG) modes caused by the presence of impurities in the edge and by the resulting core density peaking [9].
TEXTOR-94 is a medium size tokamak $(R=$ $1.75 \mathrm{~m}, a=0.46 \mathrm{~m}$ ) equipped with the toroidal belt limiter ALT-II which consists of eight pumped blades. Some blades are equipped with gas inlet apertures located at the surface of the limiter tiles. The gas flow through the ALT fueling apertures is smoother than it is for the standard TEXTOR gas feeders equipped with a fast feedback controlled gas fueling. Both systems are used successfully in this work.

Figure 1 shows an example of a RI-mode discharge where $\mathrm{H}$-mode confinement quality has been obtained at $\bar{n}_{e, 0} / n_{\mathrm{GW}}=1.4$ under quasistationary conditions with $\beta$

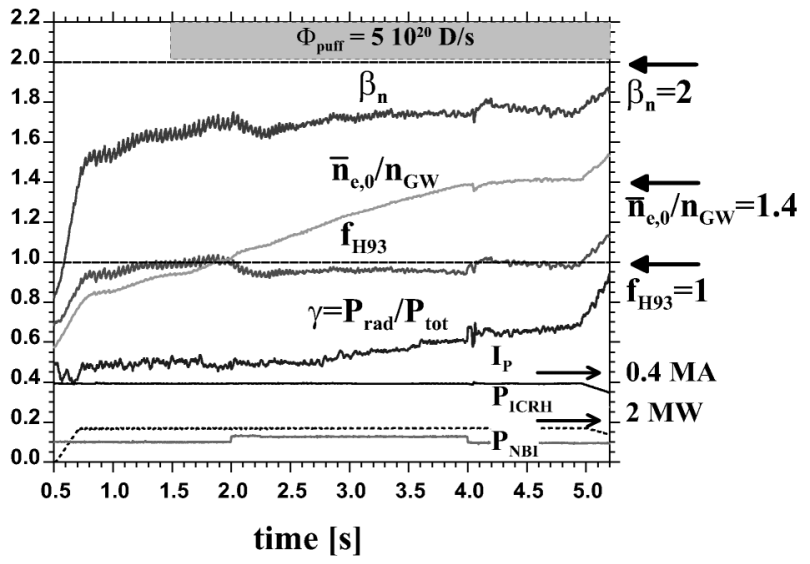

FIG. 1. Time evolution of nondimensional parameters for a discharge reaching $\bar{n}_{e, 0} / n_{\mathrm{GW}}=1.4$ with $f_{H 93}=1$ and $\beta_{n}=$ 1.8 for a stationary phase (between 4 and $5 \mathrm{~s}$ ) of $20 \tau_{E}$ up to the current ramp-down phase $\left(B_{T}=2.25 \mathrm{~T}\right.$, siliconized wall $)$ by gas fueling through a limiter blade (the injection rate $\Phi_{\text {puff }}$ is indicated in the figure). The time traces of the plasma current $I_{P}$ and of additional heating power (ICRH and NBI) are also shown. A small amount of counter-neutral-beam injection is added from 2 to $4 \mathrm{~s}$ for diagnostic purposes. 
values close to the operational limit of TEXTOR-94 ( $\beta_{n} \approx$ 2 ) for the typical RI-mode conditions [10]. The key factor to obtain these results is appropriate tailoring of the deuterium gas fueling in addition to (i) careful wall conditioning (in this case fresh siliconization) and a proper positioning of the discharge with respect to the limiters, leading to conditions which avoid multifaceted radiation from the edge (MARFE) formation at high density by reducing the recycling at the inner wall [11], and (ii) edge radiation cooling (in this case due to sputtering of $\mathrm{Si}$ ), these two last conditions being necessary for high confinement radiative mantle discharges in TEXTOR. Similar results are found in discharges with additional neon seeding to produce the radiative belt under boronized or siliconized wall conditions.

The discharge is heated by $1 \mathrm{MW}$ neutral beam coinjection $\left(P_{\mathrm{NBI}}\right)$ from 0.3 to $5.5 \mathrm{~s}$ and $1.7 \mathrm{MW}$ ion cyclotron resonance heating $\left(P_{\mathrm{ICRH}}\right)$ from 0.7 to $5.0 \mathrm{~s}$. The density rise due to gas injection through an ALT blade leads to a reduction from $Z_{\text {eff }}(0) \approx 2$ at $1 \mathrm{~s}$ to $Z_{\text {eff }}(0) \leq 1.5$ at $t>2.5 \mathrm{~s}$. During the density ramp no MARFEs appear. The discharge is initially in the RI-mode regime due to the edge radiation produced by silicon sputtering and follows the RI-mode scaling up to $\bar{n}_{e, 0}=n_{\mathrm{GW}}$. At higher densities, the confinement remains high with a quality equal to ELM-free $\mathrm{H}$-mode confinement, i.e., with a value for the enhancement factor $f_{H 93}$ with respect to the ITERH93-P scaling expression (applied to densities above $\bar{n}_{e, 0}=n_{\mathrm{GW}}$ ) equal to 1 . Furthermore the phase with $\bar{n}_{e, 0} / n_{\mathrm{GW}}=1.4$ is kept quasistationary for a duration of 20 confinement times $\left(\tau_{E}=50 \mathrm{~ms}\right)$. At the same time, the normalized $\beta_{n}$ is maintained at 1.8 , close to the beta limit of TEXTOR $\left(\beta_{n} \approx 2\right)$. The radiation fraction $\gamma=P_{\text {rad }} / P_{\text {tot }}$ (with $P_{\text {rad }}$ the radiated power and $P_{\text {tot }}$ the total applied heating power) is not very sensitive to the density due to a self-regulation of the silicon sputtering [12] and increases moderately from 0.5 to 0.7 for the highest densities reached. The phase with high density and high confinement is terminated by the preprogrammed rampdown of the plasma current and additional heating power in order to avoid hard disruptions at the end of these highdensity discharges.

The confinement characteristics of high-density discharges on TEXTOR-94 are illustrated by comparing the behavior of RI- and L-mode discharges in Fig. 2, where the normalized confinement time $\tau_{E} P_{\text {tot }}^{2 / 3} / I_{P}\left(P_{\text {tot }}\right.$ is the total heating power in MW) is plotted versus the Greenwald number $\bar{n}_{e, 0} / n_{\mathrm{GW}}$ as introduced in Ref. [3]. The data in this diagram, which have been obtained after a fresh siliconization, illustrate three types of behavior depending on the deuterium (D) gas fueling rate.

(i) The triangles correspond to RI-mode discharges with a confinement scaling given by $\tau_{\mathrm{RI}}=K \bar{n}_{e, 0} P_{\mathrm{tot}}^{-2 / 3}(K=$ 0.18 ; using s, $10^{20} \mathrm{~m}^{-3}$, and MW as units). Low D gas puffing rates or no D gas puff at all is applied in these

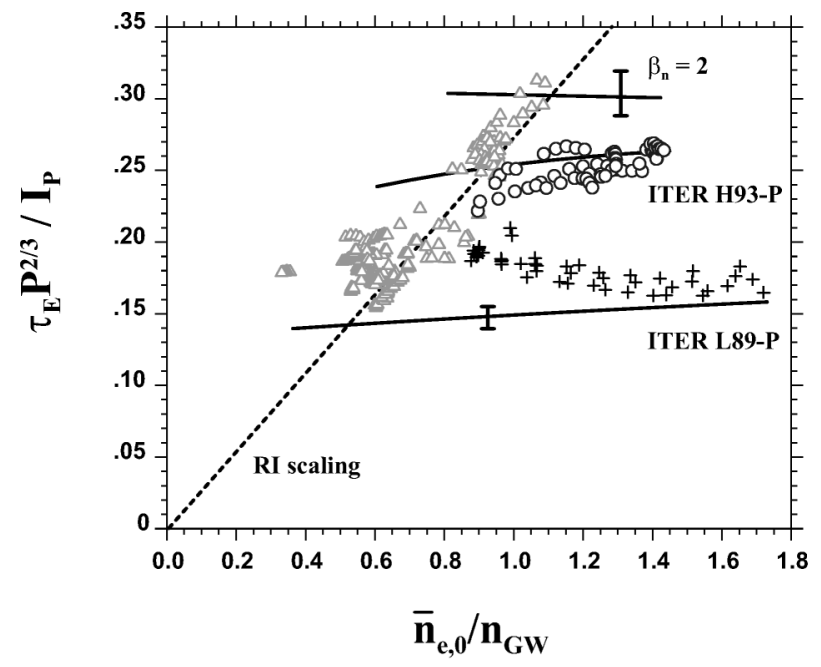

FIG. 2. Normalized diagram of $\tau_{E} P^{2 / 3} / I_{P}$ versus $\bar{n}_{e, 0} / n_{\mathrm{GW}}$ for discharges with RI-mode (triangles), L-mode (crosses), and intermediate confinement (circles). The corresponding predictions from L-mode (ITER L-89 P), H-mode (ITER H93-P), and RImode scalings are included. The location of the operational beta limit close to $\beta_{n}$ is indicated. The error bars are related to the parameter range of the experimental data.

discharges. The maximum obtainable confinement is limited by the $\beta$-limit determining the maximum achievable density compatible with the RI-mode scaling, given by $\left(\bar{n}_{e, 0} / n_{\mathrm{GW}}\right)_{\max }=\beta_{n, \text { max }} P_{\mathrm{tot}}^{-1 / 3} B_{T} /(15.9 \mathrm{~K})$ with the same units as before and the toroidal magnetic field $B_{T}$ in T. For the experimental values of $P_{\text {tot }}$ and the toroidal field $B_{T}=2.25 \mathrm{~T}$, this relation limits the maximum density for RI-mode discharges to $\left(\bar{n}_{e, 0} / n_{\mathrm{GW}}\right)_{\max } \cong 1.1$ at $\beta_{n} \approx 2$. The error bar placed on the curve indicating the $\beta$-limit in Fig. 2 reflects the variation in the applied heating power used in the experiments.

(ii) The crosses correspond to discharge conditions for which very high Greenwald numbers are reached but where the confinement is degraded down to L-mode confinement. These discharges are characterized by a strong D gas puff.

(iii) The circles correspond to discharges that follow an intermediate path between RI- and L-mode scaling and are obtained by tailoring the D gas-fueling rate in order to maintain the edge pressure sufficiently low as discussed later. This technique is applied in the discharge of Fig. 1 in order to reduce its initial RI-mode confinement to somewhat lower values, sufficient to avoid the $\beta_{n}$ limit but not too much in order to maintain a confinement with at least ELM-free H-mode quality. This result is independent of the radiating impurity and has been successfully applied with silicon radiation (under freshly siliconized conditions) or with neon radiation under freshly boronized conditions.

The decisive influence of the $\mathrm{D}$ gas-fueling rate on confinement is further illustrated in Fig. 3 where the time evolution of two discharges with freshly boronized wall and neon seeding but with different $\mathrm{D}$ gas-fueling rates are compared. These various rates have been achieved by 


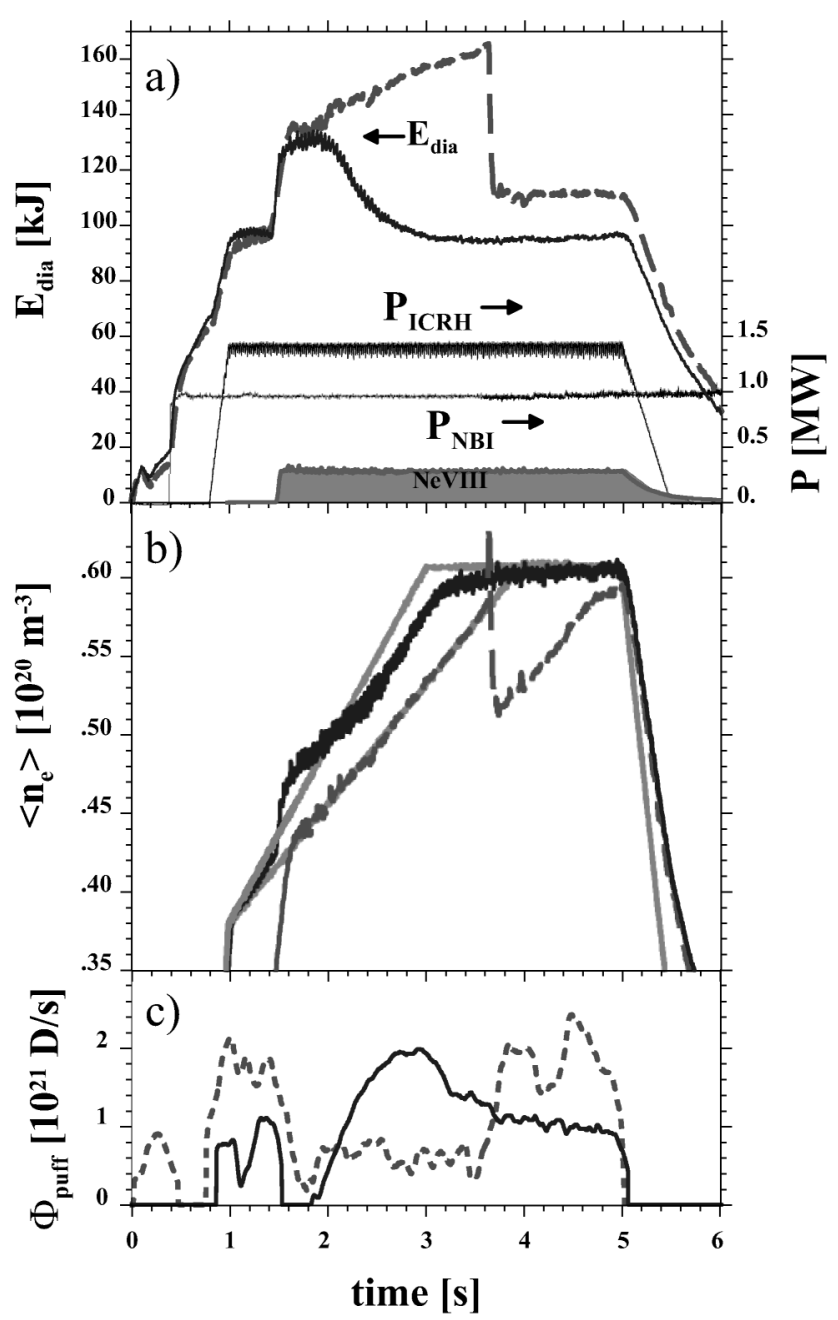

FIG. 3. (a) Comparison of the time evolution of the diamagnetic energy of two discharges with slower (dashed line) or steeper (solid line) slope of programmed value of the feedback controlled volume averaged density $\left(\left\langle n_{e}\right\rangle\right)$. The common NBI and ICRH heating powers and Ne brilliance (freshly boronized condition) are indicated. (b) Preprogrammed (in grey) and actual value of $\left\langle n_{e}\right\rangle$ (solid and dashed lines). (c) $\mathrm{D}$ gas puffing rate for the slower (dashed line) and steeper (solid line) slope of $\left\langle n_{e}\right\rangle$.

programming different density ramps in the density feedback system. Both discharges are heated by the same amount of NBI-co (starting at $t=0.4 \mathrm{~s}$ ) and ICRH (starting at $t=1 \mathrm{~s}$ ). Neon seeding starts at $t=1.4 \mathrm{~s}$. The discharges are terminated by a ramp-down of the plasma current $I_{P}$ at $t=5.0 \mathrm{~s}$. At the start of the neon seeding, a transition from L- to RI-mode occurs for both discharges characterized by an increase in the energy and particle confinement time. Consequently the $\mathrm{D}$ gas fueling rate of both discharges is reduced and the diamagnetic energy $E_{\text {dia }}$ rises strongly. For the discharge with the lower slope of the programmed density (dashed line) the diamagnetic energy increases with the density in agreement with the RI-mode scaling until the $\beta$ limit is reached at a density $\bar{n}_{e, 0} / n_{\mathrm{GW}}=1.2$. At that point magnetohydrodynamic (MHD) activity [10] causes back transition leading to a confinement quality between L- and RI-modes. The electron density also drops due to the simultaneous decrease of the deuterium particle confinement time $\tau_{p, D}$ and the density feedback control system reacts by increasing the fueling rate. This discharge scans the behavior represented by the triangles of Fig. 2. In the second discharge (solid line) the slope of the preprogrammed density ramp is higher. This leads to a stronger $\mathrm{D}$ gas puff rate than for the first discharge and confinement degrades progressively from RIto L-mode values. This behavior is the one observed for the crosses in Fig. 2.

A strong correlation is found between the edge neutral pressure $p_{n}$ (measured in the pumping duct of the ALT-II limiter blades or at the machine wall sufficiently away from the gas inlet valves, both being proportional to each other) and the confinement degradation with respect to the RI-mode scaling (expressed by the ratio $f_{h, \mathrm{R} I}$ of the experimental energy confinement time to the RImode scaling $\left.f_{h, \mathrm{RI}}=\tau_{E, \exp } / \tau_{E, \mathrm{RI}}\right)$. This is illustrated in Fig. 4 for a large set of discharges pertaining to the same machine conditions including the different types of discharges of Figs. 1 and 3. From Fig. 4 follows, remarkably, that the same degradation curve applies independently of the plasma density, as represented by the different symbols. Consequently for any density the confinement can be found by multiplying the RI-mode scaling by a degrading function $f\left(p_{n}\right)$. Thus, if the density reached by careful gas fueling is high enough, $\tau_{\mathrm{R} I}$ will exceed substantially the L-mode scaling such that despite a low value of $f\left(p_{n}\right)$ the confinement is nevertheless compatible with

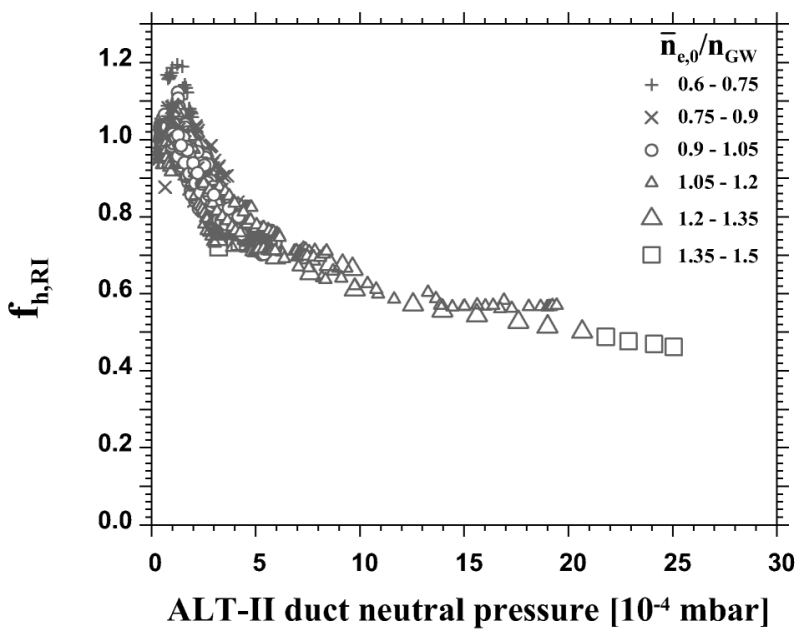

FIG. 4. Degradation factor with respect to the RI-mode confinement scaling $f_{h, \mathrm{RI}}$ as a function of the edge pressure as measured in the pumping duct of the ALT-II limiter. The different symbols correspond to different ranges of the Greenwald number. 
an H-mode confinement quality as is the case for the discharge of Fig. 1. As $f\left(p_{n}\right) \leq 1$, the RI-mode confinement is the best possible at high density in TEXTOR. A good correlation also exists between $f_{h, \mathrm{R} I}$ and the recycling flux at the main limiter ALT-II which is an increasing function of $p_{n}$.

The question that arises is to know what the causality in establishing the relationship between edge pressure and confinement is, i.e., whether the confinement degradation is leading to an increased edge pressure, or whether it is the inverse. Remarkably both causalities are possible.

(i) a confinement degradation (caused by, e.g., MHD instabilities) can cause an increase in the edge neutral density. This seems obvious due to the associated loss of particle confinement time. An example of such a case occurs at the confinement back transition illustrated in Fig. 3, and the corresponding behavior of $f_{h, \mathrm{RI}}=f\left(p_{n}\right)$ coincides with the trajectory described by the points of Fig. 4.

(ii) The inverse of (i) is illustrated in Fig. 5: a gas puff flux which is always much smaller than the recycling flux can be the cause of a confinement degradation. The $\mathrm{D}$ gas injection valve is opened at $1.7 \mathrm{~s}$, leading to an increase of the recycling flux and of the edge pressure (along the wall or in the pumping duct of the ALT-II limiter), followed about $300 \mathrm{~ms}$ later by first signs of a decrease in the confinement quality expressed by $f_{h, \mathrm{R} I}$, thus giving no doubt about the causality. The density peaking factor $\gamma_{n}=\bar{n}_{e, 0} /\left\langle n_{e}\right\rangle$ also starts to decrease before the confinement degradation. A strong correlation with the gas influxes is also seen in the D-T experiments on TFTR [13] and in the transition from saturated Ohmic confinement to improved Ohmic confinement [14].

It is worth noting that the injection of neon does not lead, at given $\bar{n}_{e, 0}$, to an increase of the edge neutral pressure, but, on the contrary, to a reduction of it due to the resulting increase of the deuterium particle confinement time $\tau_{p, D}$.

The increase of the edge pressure is linked with an increase of the edge electron density and, for a given $\bar{n}_{e, 0}$, a decrease of the density profile peaking. The steepening of the density profile is considered together with the presence of impurities at the edge to trigger the confinement improvement in the RI-mode through stabilization of the ITG-modes [9].

Other observed effects resulting from the increase of the edge neutral pressure and the resulting decrease of $f\left(p_{n}\right)$ are (i) the concomitant increase of the exhaust efficiency of the pump limiter $\left(\eta_{\text {exh }}=\Phi_{\text {pump }} / \Phi_{\text {recycling }}\right.$, with $\Phi_{\text {pump }}$ and $\Phi_{\text {recycling }}$ as pumping and recycling fluxes, respectively) and (ii) the decrease of the product of the deuterium particle confinement time $\tau_{p, D}$ and the fueling efficiency $f$ at a given $\bar{n}_{e, 0}: f \tau_{p, D}=N_{\text {tot }} / \Phi_{\text {recycling }}$ (with $N_{\text {tot }}$ the total number of particles) [15].

The effective deuterium particle confinement time $\tau_{p, D}^{*}=\tau_{p, D} /(1-R)=\tau_{p, D} / \eta_{\text {exh }} \quad$ (where $R$ is the recycling coefficient) decreases experimentally with an increasing edge pressure. The behavior of helium is similar;

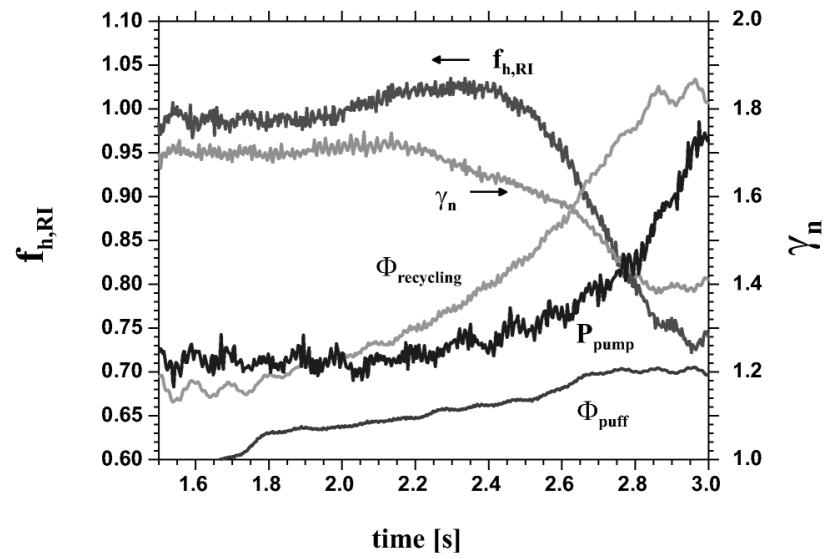

FIG. 5. Gas injection into a RI-mode discharge (siliconized wall) leading to a confinement degradation. After increasing gas injection flux $\Phi_{\text {puff }}$ (scale from 0 to $9 \times 10^{21} \mathrm{D} / \mathrm{s}$ ), starting at $1.7 \mathrm{~s}$, the edge pressure as measured in the ALT-II duct $p_{\text {pump }}$ (scale from 0 to $4 \times 10^{-4} \mathrm{mbar}$ ), and the recycling flux $\Phi_{\text {recycling }}$ (scale from 0 to $5 \times 10^{22} \mathrm{D} / \mathrm{s}$ ) increase. Afterwards the density peaking $\gamma_{n}$ decreases before the start of the confinement degradation.

i.e., an increase in the exhaust efficiency and a decrease of $\tau_{p, \mathrm{He}}^{*}$ of helium is also observed with an increasing edge pressure [and thus decreasing $f\left(p_{n}\right)$ ]. Optimized high-density discharges (as, e.g., shown in Fig. 1) can therefore maintain low values of $\rho=\tau_{p, \mathrm{He}}^{*} / \tau_{E}$ equal to about $10-15$, relevant for reactor conditions [16].

[1] M. Greenwald et al., Nucl. Fusion 28, 2199 (1988).

[2] A. M. Messiaen et al., Phys. Rev. Lett. 77, 2487 (1996).

[3] R. R. Weynants et al., Nucl. Fusion 39, 1637 (1999).

[4] M. A. Mahdavi et al., in Proceedings of the 24th European Conference on Berchtesgaden, 1997, Controlled Fusion and Plasma Physics (Institute of Particle Physics, Bristol, U.K., 1997), Vol. 21A, Pt. III, p. 1113.

[5] M. R. Wade et al., J. Nucl Mater. 266-269, 44 (1999).

[6] M. A. Mahdavi et al., Bull. Am. Phys. Soc. 44, 169 (1999); (private communication).

[7] T. Osborne et al., Bull. Am. Phys. Soc. 44, 128 (1999); (private communication).

[8] L. D. Horton et al., Nucl. Fusion 39, 1 (1999).

[9] M.Z. Tokar et al., Phys. Rev. Lett. 84, 895 (2000).

[10] H. R. Koslowski et al., Nucl. Fusion 40, 821 (2000).

[11] J. Rapp et al., Nucl. Fusion 39, 765 (1999).

[12] U. Samm et al., J. Nucl. Mater. 220-222, 25 (1995).

[13] J. Strachan et al., Nucl. Fusion 39, 919 (1999).

[14] M. Tokar, Plasma Phys. Controlled Fusion 35, 1119 (1993).

[15] B. Unterberg et al., in Proceedings of the 26th European Conference on Maastricht, 1999, Controlled Fusion and Plasma Physics (Institute of Physics Publishing, Bristol, U.K., 1999), Vol. 23J, p. 721.

[16] D. Reiter, G. H. Wolf, and H. Kever, Nucl. Fusion 30, 2141 (1990). 